\title{
FARE PRAGMATICA NELla SCUOLA PRIMARIA: UNO STUDIO ESPLORATIVO SULLE RICHIESTE
}

\author{
Stefania Ferrari* \\ Greta ZanONI*
}

\begin{abstract}
L'articolo illustra uno studio esplorativo sulle modalità di produzione dell'atto comunicativo del richiedere nell'interazione di bambini di 8-9 anni, basato su una sperimentazione educativa di ricerca-azione, condotta in Italia nella classe curricolare della scuola primaria. Gli aspetti specificamente indagati sono i seguenti: le modalità di realizzazione dell'atto comunicativo; la relazione tra atto comunicativo e sequenza interazionale; la capacità dei bambini di riflettere sugli usi linguistici. I risultati ottenuti offrono indicazioni per lo sviluppo di materiali didattici appropriati e per la definizione di futuri protocolli di ricerca.

PAROLE CHIAVE: Pragmatica; lingua italiana; scuola primaria; richieste; interazione orale.

RESUMO: $O$ artigo ilustra um estudo exploratório sobre as modalidades de produção do ato comunicativo do pedir na interação de crianças de 8-9 anos, baseado em uma experimentação educacional de pesquisa-ação, conduzida na Itália na turma curricular da escola primária. Os aspectos especificamente indagados são os seguintes: as modalidades de realização do ato comunicativo; a relação entre ato comunicativo e sequência interacional; a capacidade das crianças de refletir sobre os usos linguísticos. Os resultados obtidos oferecem
\end{abstract}

* Università degli Studi di Verona, Verona (Italia) - stefaniaferrari@tiscali.it ** Università degli Studi di Bologna, Bologna (Italia) - greta.zanoni2@unibo.it 
indicações para o desenvolvimento de materiais didáticos apropriados e para a definição de futuros protocolos de pesquisa.

PALAVRAS CHAVE: Pragmática; língua italiana; escola primária; pedidos; interação oral.

ABSTRACT: The present paper illustrates an exploratory study investigating the communicative act of request performed by children aged 8-9 in oral interactions with adults and peers. The study is based on data collected as part of an educational action research plan carried out in two classrooms at a primary school in Italy. The main aspects investigated are: the strategies for requesting in oral production; the relationship between communicative act and interactional sequence; the ability of children to reflect on the language they use. Results provide useful insights both for the development of specific teaching materials and the definition of future research protocols.

KEYWORDS: Pragmatics; Italian language; primary school; requests; oral interaction. 


\section{Introduzione}

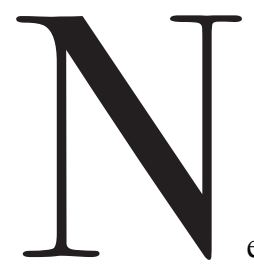

ella scuola italiana, fin dalle prime classi della primaria, i docenti di educazione linguistica manifestano spesso preoccupazione per la qualità delle competenze d'uso orale della lingua da parte dei loro allievi, siano essi studenti italofoni, bambini bilingui con una lingua familiare diversa dall'italiano o apprendenti di italiano L2. Capita, ad esempio, nell'interazione con gli insegnanti o con i pari, che l'impiego di espressioni o formule pragmaticamente inappropriate, oltre a limitare il raggiungimento dello scopo comunicativo che si era prefissato il parlante, porti a giudizi stereotipati sul singolo allievo, quando italofono, o addirittura sulla comunità di provenienza, quando non italofono. Risulta pertanto importante, nell'ambito dell'educazione linguistica, promuovere la diffusione di pratiche didattiche capaci di sostenere lo sviluppo delle abilità pragmatiche, integrandole alle altre competenze linguistiche, già oggetto di intervento didattico nelle ore dedicate alla lingua italiana. D'altronde, chi si occupa di apprendimento linguistico sa bene che imparare una lingua, sia 
essa L1 o L2, non significa solo acquisire lessico e grammatica, ma anche sviluppare quella serie di abilità che permettono di mettere in relazione parole e contesto comunicativo, guidando il parlante sia nella scelta delle formulazioni linguistiche più adatte alle specifiche situazioni sia nell'interpretazione delle eventuali sfumature di senso o dei significati impliciti delle parole usate (BAZZANELLA, 1994, 2005; BETTONI, 2006; ANDORNO, 2005; NUZZO, 2007).

Nonostante si riconosca in letteratura il bisogno di rivolgere un'attenzione didattica specifica allo sviluppo della abilità d'uso della lingua (cfr. NUZZO; GAUCI, 2012; SANTORO; VEDDER, 2016), risultano ancora carenti (se non assenti), per l'italiano L1 e L2, studi e materiali dedicati alla fascia d'età della scuola primaria. Le ricerche che indagano l'acquisizione delle competenze pragmatiche si rivolgono a bambini in età prescolare e riguardano altre lingue, come ad esempio l'inglese (cfr. MATTHEWS, 2014) o, quando dedicati all'italiano, si basano su dati relativi ad adolescenti e adulti (cfr. NUZZO; GAUCI, 2012; SANTORO; VEDDER, 2016). Anche i materiali didattici a disposizione sono rari e, nel caso dell'italiano, pensati esclusivamente per adulti che apprendono l'italiano come L2. Gli stessi manuali per stranieri rappresentano una fonte di input pragmatico tendenzialmente povera (NUZZO, 2016), sebbene sia possibile indicare qualche recente eccezione (cfr. Progetto LIRA, http://lira.unistrapg.it; FERRARI et al., 2016).

Nella pratica didattica a scuola, sia i testi scolastici per la classe curricolare che i manuali di L2 per le attività laboratoriali, si occupano principalmente di insegnamento di lessico e grammatica. La riflessione linguistica in L1 oltre a essere dedicata principalmente alla lingua scritta, con le abilità del parlato di rado contemplate nell'insegnamento in aula (cfr. LEONE; MEZZI, 2011), è intesa quasi esclusivamente come analisi grammaticale, logica e del periodo (MIGLIETTA; SOBRERO, 2011). Ne consegue che, anche per assenza di stimoli provenienti dai materiali in uso, difficilmente il docente addestra gli allievi, in maniera consapevole e sistematica, a fare cose con le parole.

L'insegnante che vuole far spazio alla pragmatica nell'ora di educazione linguistica si trova nella condizione di dover individuare - senza poter far affidamento su indicazioni precise provenienti, appunto, dai sussidi didattici - strategie pedagogiche specifiche che permettono una fluida integrazione tra insegnamento di lessico, grammatica e sviluppo delle competenze pragmatiche, oltre che di dover offrire stimoli adeguati per una ricchezza di contesti comunicativi in cui sia possibile sperimentare la variazione linguistica rispetto agli usi orali e scritti della lingua.

Poiché non è possibile perseguire un tale obiettivo educativo senza avere accesso a conoscenze teoriche e strumenti operativi che permettono di affrontare in modo consapevole l'insegnamento della pragmatica, a partire dall'anno scolastico 2015/2016 si è dato il via al percorso di formazione e ricerca-azione Oggi facciamo pragmatica. Alla base del progetto vi è l'idea che l'insegnamento della pragmatica non solo possa iniziare fin dalla scuola primaria, ma debba essere opportunamente integrato nella quotidianità dell'educazione linguistica, in un percorso in cui grammatica e pragmatica dialogano tra loro, dove le nozioni linguistiche 
vengono riprese nella pratica d'uso e, viceversa, a partire dall'uso della lingua si riattiva la riflessione grammaticale (FERRARI, 2016).

La ricerca-azione, fin dal suo primo anno di sperimentazione, oltre ad aver prodotto interventi di educazione linguistica per le classi, ha permesso la raccolta di un corpus di produzioni orali di bambini di età compresa tra gli 8 e i 9 anni, costituito da interazioni contenenti una varietà di atti comunicativi di richieste e da commenti meta-pragmatici stimolati durante le attività dedicate alla riflessione linguistica. Tali dati sono alla base della ricerca presentata in questo contributo. Nei paragrafi che seguono si illustrano in dettaglio il contesto dello studio esplorativo ( $\$ 2$ ), l'analisi quantitativa degli atti comunicativi di richiesta ( $\$ 3$ ) e l'analisi qualitativa dei commenti meta-pragmatici emersi nelle attività di riflessione linguistica $(\$ 4)$. Si conclude con alcune indicazioni per l'elaborazione di materiali didattici e per la definizione di futuri protocolli di ricerca $(\S 5)$.

\section{Lo studio esplorativo}

Questo lavoro nasce con lo scopo di indagare come viene utilizzata la lingua italiana da parte di bambini della scuola primaria in alcune situazioni di richiesta legate ad ambiti di esperienza quotidiani, nell'interazione con pari o con adulti.

Quattro sono le domande che stanno alla base del presente studio esplorativo:

a. Come realizzano l'atto comunicativo di richiesta i bambini della scuola primaria?

b. Come si sviluppa la richiesta nella sequenza interazionale?

c. Come rispondono i bambini alle attività didattiche proposte?

d. Che tipo di riflessioni meta-pragmatiche fanno gli allievi di questa età?

Individuate le finalità generali della ricerca, si è proceduto con la raccolta di un corpus di dati. Poiché non è semplice, con bambini di questa fascia di età, stimolare produzioni linguistiche $a d$ hoc, mantenendo una certa validità ecologica si è ritenuto efficace integrare la raccolta di dati con la sperimentazione educativa nell' ambito del progetto di ricerca-azione Oggi facciamo pragmatica. In particolare si è fatto riferimento alle sperimentazioni del percorso didattico relativo all'atto comunicativo del richiedere realizzato rispettivamente in una classe terza e in una classe quarta della scuola primaria. Gli informanti coinvolti sono 51 allievi, di cui 33 monolingui italiani e 18 bilingui con una lingua familiare diversa dall'italiano, tutti scolarizzati in Italia.

Il percorso didattico (cfr. FERRARI, 2016 per una descrizione dettagliata) ha avuto una durata complessiva di circa 6 ore, distribuite su 3-4 lezioni, secondo una struttura articolata in 4 fasi: 1 -Osservare; 2 -Utilizzare; 3 -Riflettere; e infine 4 -Fare esperienza. 
La fase 1 - Osservare - aveva lo scopo di presentare agli alunni l'atto comunicativo trattato nel percorso, oltre a offrire occasione al docente di classe di valutare che cosa gli allievi sapevano già fare rispetto agli obiettivi dell'intervento; la fase 2 - Utilizzare - metteva gli studenti nella condizione di usare la lingua in specifici contesti comunicativi stimolati a partire da role play aperti spontanei, mirati a elicitare l'atto del richiedere; la fase 3 - Riflettere - era il momento della riflessione linguistica e grammaticale, condotta attraverso attività di analisi e di pratica per il reimpiego delle strutture target; infine, la fase 4 - Fare esperienza - era dedicata all'osservazione della lingua in contesti d'uso reali, permettendo così di favorire un ponte tra esercitazione in aula e vita reale.

A partire dalla video-registrazione delle lezioni è stato possibile costruire un corpus di produzioni degli alunni delle due classi composto da due tipologie di dati: la prima, costituita da interazioni orali contenenti atti comunicativi di richiesta elicitati tramite role play aperti spontanei; la seconda, da una selezione di commenti meta-pragmatici generati dalle attività didattiche di riflessione linguistica.

Rispetto alla prima tipologia di dati, i role play hanno permesso di stimolare 81 scambi interattivi contenenti altrettanti atti comunicativi di richiesta: 39 relativi alla richiesta di beni materiali, di cui 31 rivolti a pari e 8 ad adulti, e 42 riguardanti richieste di permessi rivolte principalmente ad adulti, con un solo esempio di richiesta a un pari. Ogni role play prevedeva la presenza di due interlocutori, ed è stato quindi interpretato a coppie. A ciascun bambino veniva distribuito un cartoncino contenete la descrizione scritta della situazione proposta dal proprio punto di vista e veniva invitato a immedesimarsi in tale situazione e prepararsi per metterla in scena insieme al compagno davanti alla classe. Nei casi invece in cui si prevedeva un interlocutore adulto, per evitare ruoli eccessivamente innaturali, questo veniva interpretato dal ricercatore, con le istruzioni pertanto riferite solo al bambino. Ecco un esempio per ciascuno dei due tipi di consegne:

\section{Situazione tra pari: la colla}

Bambino A:

Come al solito hai dimenticato la colla a casa e la maestra vi ha appena chiesto di incollare delle immagini sul quaderno. Chiedi la colla al tuo compagno.

Bambino B:

Siete in classe e state incollando delle immagini sul quaderno. Hai poca colla e speri il tubetto non finisca prima di aver finito il compito assegnato.

Situazione adulto-bambino: il compleanno

Sabato è il tuo compleanno e hai deciso di fare una piccola festa in casa. Vuoi invitare anche il tuo amico/la tua amica. Sei all'uscita della scuola e chiedi a sua madre di lasciarlo/lasciarla venire alla tua festa 
Nella preparazione delle consegne dei role play si sono selezionate situazioni comuni relative all'esperienza quotidiana dei bambini e ai loro effettivi bisogni comunicativi. Nelle istruzioni non si è mai fatto riferimento esplicito all' atto comunicativo che si intendeva elicitare, in modo che i parlanti potessero reagire in modo spontaneo allo scambio comunicativo. Durante lo svolgimento del role play non si ponevano limiti al numero e alla lunghezza dei turni, che dipendevano esclusivamente dai tempi di raggiungimento dell'obiettivo conversazionale, così come esemplificato nell'estratto che segue:

(1) B: ciao
A: ciao dimmi
B: stamattina:: non ho mangiato ho fame \#0_2 mi potresti preparare un un biscotto?
A: certo
B: grazie

Rispetto alla seconda tipologia di dati, le attività didattiche di riflessione linguistica hanno generato sia sequenze di interazione docente-alunni contenenti commenti meta-pragmatici relativi alle interazioni nei role play della fase 2 , sia sequenze con riflessioni stimolate durante le attività di riflessione linguistica della fase 3 del percorso didattico sperimentale. Più in dettaglio, nella fase 2 - Utilizzare, immediatamente dopo la realizzazione di ciascun role play, ai bambini veniva richiesto di individuare alcune variabili situazionali, quali 'il contesto' e 'gli interlocutori', oltre che di ripetere con precisione l'esatta formulazione linguistica della richiesta. Seguiva poi un momento di analisi di gruppo, mediata dal docente sperimentatore, che permetteva ai bambini di prestare attenzione al rapporto tra strategie e forme linguistiche utilizzate, effetto sull'interlocutore ed efficacia dell'azione linguistica. Nella fase 3 Riflettere invece le attività di riflessione linguistica proposte agli allievi, da svolgere in parte individualmente, in parte a coppie, venivano strutturate utilizzando campioni di lingua emersi nei role play, con un'attenzione specifica all'analisi e al reimpiego di strutture linguisticogrammaticali funzionali a variare il tipo di atto, mitigarne la forza illocutoria o introdurlo con atti di supporto.

Infine, ai dati del corpus raccolti durante le attività didattiche in aula si aggiungono le riflessioni dei docenti emerse negli incontri di formazione dedicati alla condivisione sulle diverse fasi della sperimentazione in aula dei percorsi didattici progettati insieme al formatore.

Per rispondere alle domande di ricerca si è proceduto con due tipi di analisi, la prima quantitativa e la seconda qualitativa. Nello specifico, l'analisi quantitativa ha riguardato le produzioni elicitate tramite role play con l'obiettivo di indagare come i bambini realizzano le richieste e come le gestiscono nell'interazione. Per descrivere le modalità di realizzazione dell'atto comunicativo si è calcolato: a) il numero e il tipo di atti linguistici di richiesta, di modificatori e di atti di supporto; b) la loro distribuzione nei due tipi di role play del corpus - richieste di beni materiali vs permessi - e rispetto all'interlocutore - pari vs adulto -. Per 
osservare la relazione tra atto comunicativo e sequenza interazionale si è invece considerato: a) il numero complessivo di interazioni in cui l'atto di richiesta veniva realizzato su più turni; b) la distribuzione dell'atto nella sequenza interazionale. L'analisi qualitativa ha riguardato invece le sequenze di interazione docente-alunni relative alle attività di riflessione linguistica previste dal percorso didattico, con l'obiettivo di valutare la risposta degli allievi alle proposte didattiche e osservare il tipo di riflessioni meta-pragmatiche che è possibile stimolare in questa fascia di età.

\section{Analisi quantitativa}

\subsection{Categorie d'analisi}

Per l'analisi delle interazioni elicitate tramite role play si è scelta come unità di riferimento l'atto comunicativo, un'unità più ampia che può essere costituita da un solo atto linguistico o da una combinazione di atti, anche dotati di una forza illocutoria differente, ed eventualmente integrata da atti di supporto che hanno la funzione di sostenere o preparare la richiesta, ma che sono appunto interpretabili tenendo conto dell'unità più ampia dell'atto comunicativo, che ne garantisce la coerenza pragmatica (cfr. OLSHTAIN; COHEN, 1983, p.18-20; FERRARA, 1985, p.141; TROSBORG, 1995, p.145-6). L'atto comunicativo del richiedere ha dunque un nucleo costituito da uno o più atti, ciascuno dei quali potrebbe essere sufficiente per la trasmissione della sua principale forza illocutoria e può essere completato da atti di supporto, i quali non sono però necessari per la trasmissione della forza illocutoria, ma piuttosto hanno la funzione di preparare e sostenere la comunicazione di tale forza. Come suggerito anche da Nuzzo (2007), l'atto comunicativo è più adatto a descrivere ciò che effettivamente avviene nella conversazione, dove i parlanti, per ottenere ciò di cui hanno bisogno, possono seguire diverse strade più o meno elaborate. Ad esempio possono scegliere se impiegare uno o più atti, variarne il tipo, accompagnarli o meno con atti di supporto e, eventualmente, modificarne la forza illocutoria mediante l'impiego di modificatori linguistici di tipo morfosintattico, lessicale o discorsivo, oltre che variare la prospettiva di realizzazione di ogni atto. Chiaramente tutti questi elementi sono strettamente interdipendenti e concorrono in combinazione a modulare la forza illocutoria dell'atto. Ai fini della ricerca, per descrivere e quantificare la presenza e l'eventuale combinazione di questi elementi, diventa necessario analizzarli separatamente, con la consapevolezza che ciò rappresenta una semplificazione.

Nell'individuare gli strumenti di analisi ci siamo rifatti alla tassonomia elaborata da Nuzzo (2007). L'autrice ha individuato e categorizzato i tipi di atti comunicativi, di modificatori e di atti di supporto sulla base di un corpus di italofoni nativi adulti. Nonostante la stessa Nuzzo (2007, p.60) segnali che la sua classificazione non intende esaurire la gamma di possibili 
strategie a disposizione dei parlanti per realizzare le richieste, per i nostri propositi si è mostrata più che adeguata. Non sorprende che nel corpus di bambini della primaria la gamma di opzioni riscontrate sia più limitata rispetto a quello dei nativi adulti: in dettaglio, per quel che riguarda $i$ modificatori solo 4 tipi sono presenti, rispetto ai 13 individuati nella tassonomia di riferimento; analogamente per gli atti di supporto solo due tipi sono rappresentati - Appello e Rabbonitore - rispetto ai 5 identificati da Nuzzo. Le categorie, tuttavia, risultano significative sia nei tipi di formulazione linguistica che nella loro distribuzione. Nelle tabelle 1, 2, e 3 che seguono si riportano le categorie emerse dall'analisi dei dati, accompagnate da esempi tratti dal corpus di questo studio.

\begin{tabular}{l|l|l}
\hline \multicolumn{2}{c}{ Tab. 1: Modi per richiedere } \\
\hline $\begin{array}{l}\text { Verifica delle condizioni } \\
\text { preparatorie }\end{array}$ & $\begin{array}{l}\text { Posso disegnare } \\
\text { con i colori? }\end{array}$ & $\begin{array}{l}\text { L'interlocutore si informa sulle possibilità di } \\
\text { ottenere ciò che desidera dall'interlocutore }\end{array}$ \\
\hline Conferma & $\begin{array}{l}\text { Mi presti la } \\
\text { colla? }\end{array}$ & $\begin{array}{l}\text { Il parlante pone all'interlocutore una domanda } \\
\text { servendosi del verbo che lessicalizza l'azione } \\
\text { richiesta }\end{array}$ \\
\hline Giustificazione & $\begin{array}{l}\text { Ho dimenticato } \\
\text { la colla }\end{array}$ & $\begin{array}{l}\text { Il parlante espone il motivo per cui si trova } \\
\text { nelle condizioni di richiedere qualcosa } \\
\text { all'interlocutore }\end{array}$ \\
\hline Desiderio & $\begin{array}{l}\text { Voglio disegnare } \\
\text { alla lavagna }\end{array}$ & $\begin{array}{l}\text { Ciò che il parlante vuole ottenere } \\
\text { dall'interlocutore è espresso nei termini di un } \\
\text { desiderio }\end{array}$ \\
\hline Ordine & $\begin{array}{l}\text { Dammi la colla } \\
\text { Il parlante utilizza la forma imperativa } \\
\text { presentando l'azione richiesta come un ordine }\end{array}$ \\
\hline Necessità & $\begin{array}{l}\text { Puoi chiedere } \\
\text { a tua madre } s e \\
\text { possiamo avere } \\
\text { qualcosa da } \\
\text { mangiare? }\end{array}$ & $\begin{array}{l}\text { Il parlante presenta l'oggetto della richiesta } \\
\text { come un evento ipotetico, che se si realizzasse, } \\
\text { avrebbe per lui conseguenze positive. In genere } \\
\text { tali conseguenze sono lasciate inespresse. }\end{array}$ \\
\hline Ho fame & $\begin{array}{l}\text { Il parlante esprime la richiesta nei termini di } \\
\text { una necessità }\end{array}$ \\
\hline Fotesi
\end{tabular}

Fonte: adattato da NUZZO (2007, pp.60-4) 


\begin{tabular}{l|l|l}
\hline \multicolumn{2}{c}{ Tab. 2: Modificatori } \\
\hline Condizionale & $\begin{array}{l}\text { Andrea potrebbe venire al mio } \\
\text { compleanno? }\end{array}$ & $\begin{array}{l}\text { Associato ad altre strategie, pone in } \\
\text { discussione l'appartenenza di quanto detto } \\
\text { alla realtà }\end{array}$ \\
\hline Rafforzatori & $\begin{array}{l}\text { Maestra, ho tanto mal di } \\
\text { pancia potrei andare in bagno? }\end{array}$ & $\begin{array}{l}\text { Accrescono il peso delle ragioni che } \\
\text { inducono il parlante a fare la richiesta }\end{array}$ \\
\hline \multirow{2}{*}{ Attenuatori } & $\begin{array}{l}\text { Maestra, possiamo solo } \\
\text { per questa volta giocare a } \\
\text { pallasqualo? }\end{array}$ & $\begin{array}{l}\text { Tendono ad alleggerire il peso dell'enunciato } \\
\text { facendo riferimento alla quantità o alla } \\
\text { qualità dell'oggetto in questione o al tempo e } \\
\text { allo sforzo richiesto all'interlocutore }\end{array}$ \\
\hline \multicolumn{3}{c}{ Discorsivi } \\
\hline cortesia & $\begin{array}{l}\text { Per favore Paola, mi puoi dare } \\
\text { metà della tua pizza? }\end{array}$ & $\begin{array}{l}\text { Mostrano l'atteggiamento di deferenza } \\
\text { del parlante e invocano la collaborazione } \\
\text { dell'interlocutore }\end{array}$ \\
\hline
\end{tabular}

\begin{tabular}{l|l|l}
\hline \multicolumn{3}{c}{ Tab. 3: Atti di supporto } \\
\hline \multirow{3}{*}{ Appello } & $\begin{array}{l}\text { Scusi maestra posso } \\
\text { ripassare per la } \\
\text { verifica? }\end{array}$ & $\begin{array}{l}\text { Richiama l'attenzione dell'interlocutore prima } \\
\text { della formulazione della richiesta e costituisce } \\
\text { spesso l'apertura dell'atto comunicativo }\end{array}$ \\
\hline Rabbonitore & $\begin{array}{l}\text { Ti prego possiamo } \\
\text { giocare? }\end{array}$ & $\begin{array}{l}\text { Sottolinea la consapevolezza, da parte del } \\
\text { parlante, della natura impositiva della richiesta, } \\
\text { invocando un atteggiamento collaborativo da parte } \\
\text { dell'interlocutore }\end{array}$ \\
\hline \multicolumn{2}{c}{ Fonte: adattato da NUZZO (2007, p.68) }
\end{tabular}

Poiché i dati del corpus sono costituiti da interazioni tra due parlanti, l'atto comunicativo può essere compiuto in un unico turno o su più turni. Tale distribuzione nella sequenza interazionale può riguardare la sola preparazione del nucleo, così come la sua presentazione: la scelta dipende sia dalla pianificazione del richiedente, che dalla reazione dell'interlocutore, poiché l'atto comunicativo è il risultato di una interazione e co-costruzione da parte dei partecipanti allo scambio comunicativo. Tenuto conto della varietà di possibili configurazioni a disposizione dei parlanti, ai fini dell'analisi qui condotta, si è stabilito di isolare e analizzare 
in dettaglio soltanto il turno (o i turni) contenente il nucleo della richiesta. Per l'individuazione si è scelto il riconoscimento della forza illocutoria da parte dell'interlocutore manifestata per mezzo della prima accettazione o rifiuto della richiesta. Consapevoli che tale taglio costituisca una forzatura, la scelta è stata resa necessaria dal bisogno di focalizzare l'attenzione sulla realizzazione degli atti comunicativi, piuttosto che sulla gestione di intere sequenze conversazionali. L'osservazione comunque si è estesa successivamente agli eventuali turni che precedono e preparano quello contenente il nucleo dell'atto, permettendo di completare l'analisi dell'atto comunicativo per sé con rilevazioni relative alla gestione dello stesso all'interno dello scambio comunicativo.

\subsection{Risultati dell' analisi quantitativa}

L'analisi quantitativa offre una visione globale delle linee di tendenza riscontrabili nel corpus in merito alle modalità di realizzazione dell' atto comunicativo di richiesta da parte di bambini di 8-9 anni impegnati in interazioni orali. Date le dimensioni del campione, non è possibile formulare generalizzazioni, che rischierebbero di essere poco attendibili, ma piuttosto identificare aspetti da indagare ulteriormente o da trattare in modo mirato in futuri interventi didattici. Vediamo in dettaglio cosa emerge dalla lettura quantitativa dei dati.

La prima analisi è relativa alla composizione del nucleo dell'atto di richiesta. Come già specificato, per la lettura dei dati si è proceduto con il conteggio del numero e del tipo di atti di richiesta, oltre che il numero e il tipo di combinazioni più frequenti. Le tabelle 4 e 5 riassumono i dati che si vanno ora a descrivere.

\begin{tabular}{l|c|c|c|c}
\hline \multicolumn{5}{c}{ Tab. 4: Composizione del nucleo dell'atto comunicativo } \\
\hline & Totale & $\begin{array}{l}\text { Beni materiali } \\
\text { pari }\end{array}$ & Beni materiali adulto & $\begin{array}{l}\text { Permesso } \\
\text { adulto }\end{array}$ \\
\hline $\begin{array}{l}\text { Nucleo realizzato con } \\
\text { un atto }\end{array}$ & $70 \%(57)$ & $52 \%(15)$ & $80 \%(8)$ & $83 \%(34)$ \\
\hline $\begin{array}{l}\text { Nucleo realizzato con } \\
\text { due atti }\end{array}$ & $26 \%(21)$ & $48 \%(14)$ & $10 \%(1)$ & $15 \%(6)$ \\
\hline $\begin{array}{l}\text { Nucleo realizzato con } \\
\text { tre atti }\end{array}$ & $4 \%(3)$ & & $10 \%(1)$ & $2 \%(1)$ \\
\hline Totale & $100 \%(81)$ & $100 \%(29)$ & $100 \%(10)$ & $100 \%(41)$ \\
\hline
\end{tabular}


Considerando il corpus nel suo complesso, gli informanti nel $70 \%$ dei casi realizzano il loro scopo comunicativo con un solo atto, mentre nel $26 \%$ dei casi il nucleo si realizza combinando due atti e solo nel $4 \%$ con tre. Disaggregando i dati per tipologia di richiesta e di interlocutore, tale tendenza viene confermata quando la richiesta, sia essa di un bene materiale (es. 2) o di un permesso (es. 3), viene rivolta ad un adulto, mentre sembra esservi un maggior uso di richieste composte di due atti quando l'interlocutore è un pari (es. 4). In quest'ultimo caso infatti nel $52 \%$ dei casi il nucleo dell'atto comunicativo è realizzato con un solo atto e il $48 \%$ dei casi con due atti.

(2) vorrei un libro di tutte le cose \#0_2 degli asteroidi del corpo umano di tutto

(3) può oggi Matteo venire a casa mia

(4) ho dimenticato la colla \#0_1 scuola \#0_2 per favore Alex me la presti

In altre parole, se nella maggior parte dei casi i bambini di questa età realizzano la richiesta in modo semplice, quando devono chiedere un oggetto in prestito al compagno, tendono a elaborarla maggiormente, combinando più frequentemente due atti, di cui uno, nella maggior parte dei casi, è di Giustificazione. Come esemplificato in (4), nel chiedere la colla al compagno il parlante prima giustifica la richiesta motivandola con una dimenticanza, mitiga l'atto con la marca di cortesia per favore, lo accompagna con un atto di supporto di Appello per richiamare ulteriormente l'attenzione del compagno, per poi esplicitare la richiesta con un atto di Conferma.

Conteggiando i tipi di atti di richiesta impiegati, così come riportato nella tabella 5, possiamo notare come i bambini impieghino tutti i tipi identificati da Nuzzo (2007). In questa fascia di età c'è dunque già una buona competenza nella variazione delle modalità di formulazione della richiesta. In linea con quanto osservato negli informanti adulti, due tipi di atti sono usati più frequentemente di altri: Verifica - impiegata nel 56,4\% dei casi - e Giustificazione - nel $21,3 \%$ dei casi -, con il primo che compare sia da solo che in combinazione con altre strategie, e il secondo che compare solo in combinazione con altre strategie. Anche disaggregando i dati per tipo di richiesta o di interlocutore, questa tendenza generale rimane confermata, cioè Giustificazione e Verifica rimangono sempre gli atti più comuni. 
Tab. 5 : Distribuzione dei tipi di atto che compongono il nucleo dell'atto comunicativo

\begin{tabular}{l|c|c|c} 
Verifica & \multicolumn{1}{|l|}{$\begin{array}{l}\text { Occorrenze } \\
\text { complessive }\end{array}$} & $\begin{array}{l}\text { Occorrenze come } \\
\text { unica strategia }\end{array}$ & \multicolumn{2}{l}{$\begin{array}{l}\text { Occorrenze in com- } \\
\text { binazione con altre } \\
\text { strategie }\end{array}$} \\
Giustificazione & $56,4 \%(61)$ & 46 & 15 \\
Conferma & $21,3 \%(23)$ & & 23 \\
Desiderio & $11,1 \%(12)$ & 5 & 7 \\
Ordine & $4,6 \%(5)$ & 4 & 1 \\
Necessità & $3,7 \%(4)$ & 4 & 2 \\
Ipotesi & $1,9 \%(2)$ & 1 & 48 \\
\hline \multicolumn{1}{c}{ Totale } & $0,9 \%(1)$ & 60 & 2 \\
\hline
\end{tabular}

Conteggiando le combinazioni più frequenti dei diversi atti emerge come Giustificazione + Verifica (es. 5) sia la strategia più diffusa con 15 occorrenze complessive - di cui 2 incluse in combinazioni di 3 atti: Giustificazione + Verifica + Desiderio e Giustificazione + Necessità + Verifica -, seguita da Giustificazione + Conferma (es. 6) con 7 occorrenze complessive.

(5) scusi maestra \#0_4 posso ripassare che \#0_2 per la verifica

(6) blea questa merenda è una schifezza \#0_2 mi dai un pezzo della tua pizza

La seconda analisi condotta sui dati riguarda la modificazione della forza illocutoria dell'atto (Tabella 6).

\begin{tabular}{l|c|c|c|c}
\hline \multicolumn{5}{c}{ Tab. 6 - Modificatori } \\
\hline & Totale & Beni materiali pari & Beni materiali adulto & Permesso adulto \\
\hline Morfosintattici & 19 & 5 & 4 & 10 \\
\hline Lessicali & 14 & 9 & 3 & 2 \\
\hline Discorsivi & 7 & 4 & & 1 \\
\hline Totale & 40 & 18 & 7 & 13 \\
\hline
\end{tabular}

Gli atti che costituiscono il nucleo di richiesta sono interessati da tale fenomeno in poco meno della metà dei casi: su 81 atti comunicativi, solo 33 sono modificati, di cui 7 con combinazione di 2 modificatori, per un totale di 40 modificatori impiegati. I modificatori morfosintattici (es. 7) come tipologia risultano i più usati e rappresentano poco meno della metà del numero 
complessivo di modificatori. Questo primo dato quantitativo è nuovamente in linea con quanto riscontrato da Nuzzo (2007). Ciò che varia negli informanti di 8-9 anni è il fatto che essi si limitano a impiegare un solo tipo di modificatore morfosintattico, il condizionale. Non vi sono nel corpus occorrenze di altre tipologie di modificatori morfosintattici (quali ad esempio negazione dell'interrogativa, imperfetto o incassatura). In modo analogo, i modificatori lessicali si confermano, così come per lo studio di Nuzzo, per frequenza d'uso il secondo gruppo per consistenza d'uso, ma di nuovo è limitata la varietà di tipi di modificatori lessicali impiegati, con soli due tipi rappresentati. Nelle produzioni dei bambini ci sono 12 occorrenze di attenuatori (es. 8), 2 di rafforzatori (es. 9), ma non vi sono casi di dubitatori, blanditori o autoumiliatori. La stessa tendenza è identificabile per i modificatori discorsivi (es. 10), rappresentati esclusivamente dalle marche di cortesia per favore, per piacere, di nuovo senza esempi delle altre tipologie (riempitivi, fatismi o richieste di accordi).

(7) scusi potrebbe venire Vivienne a casa mia

(8) maestra, ho tanto mal di pancia posso andare in bagno

(9) maestro possiamo solo per questa volta possiamo giocare a palla squalo

(10) per favore mamma di Alex ehm \#0_8 sabato ehm fai venire \#0_2 lasciate Alex alla mia festa di compleanno

L'analisi mostra dunque come i bambini di questa fascia di età riescano a produrre richieste abbastanza raffinate, modificandone la forza illocutoria, ma abbiano ancora bisogno di espandere la gamma d'uso di modificatori, variandone maggiormente il tipo.

La terza analisi condotta riguarda il numero e il tipo di atti di supporto impiegati e la loro distribuzione nel flusso conversazionale. Nel presentare i dati distinguiamo l'uso di atti di supporto all'interno e all'esterno del turno contenente il nucleo dell'atto comunicativo di richiesta. Su 81 atti comunicativi, 28 sono accompagnati da un atto di supporto all'interno del turno, mentre in soli 5 casi ciò avviene in un turno distinto. In 23 casi l'atto è rappresentato da un Appello (come nell'es. 11), mentre nei restanti 4 casi l'atto di supporto è rappresentato da un Rabbonitore (es. 12):

(11) mamma potremmo avere qualche pezzo di pane \#0_2 con la Nutella sopra

(12) ti prego \#0_4 possiamo giocare a: pallasqualo

Risultano invece rari, con 5 casi su 28, gli atti di supporto compiuti dal parlante in turni precedenti o successivi, ovvero nella fase di preparazione e di negoziazione della richiesta. Se nel corpus di NUZZO nel $20 \%$ dei casi il turno di richiesta è preceduto da un momento di "preparazione del terreno" o di ricerca della collaborazione dell'interlocutore, nel corpus di produzioni di bambini tale fase è quasi assente. Essi tendono infatti a compattare la richiesta in 
un unico turno, anche quando l'atto è elaborato in modo relativamente complesso, come negli esempi che seguono:

(13) Pietro mi presti la colla che l'ho dimenticata \#0_2 a casa

(14) Doah potrebbe venire a casa mia che: faccio una festicciola per il mio compleanno

In (13) la richiesta di un bene a un pari viene presentata combinando un atto di supporto, con l'Appello Pietro, a un atto comunicativo composto da due tipi di atti mi presti la collaConferma - che l'ho dimenticata a casa - Giustificazione, il tutto compattato in unico turno discorsivo. Analogamente in (14) il bambino si rivolge all'adulto, il genitore dell' amico, per chiedere il permesso di invitare l'amica alla sua festa di compleanno, senza introdurre la richiesta con atti di supporto, ma di nuovo condensando i due atti, rispettivamente di Verifica e di Giustificazione, con i relativi modificatori, il morfosintattico potrebbe e il lessicale festicciola, in un unico turno. I bambini dunque sono in grado di usare alcune modalità elaborate nella gestione della richiesta, ma sembrano meno capaci di gestire le richieste all'interno del flusso interazionale. Se gli adulti nativi presentano la richiesta gradualmente, diluendola su più turni dalla bassa densità informativa, i bambini condensano l'atto in un unico turno, con una presentazione esplicita e immediata della richiesta stessa. Tale differenza riflette quanto rilevato anche in altri studi, come ad esempio quelli basati sul corpus VIP, che raccoglie produzioni longitudinali di adolescenti italiani e apprendenti di italiano L2 (PALLOTTI et al., 2010). Pallotti, Ferrari (2008), Ferrari (2009; 2012), Ferrari, Nuzzo (2011) e Nuzzo (2012) evidenziano come anche le apprendenti adolescenti di italiano L2, a differenza delle compagne native, quando impegnate in richieste telefoniche, tendono a condensare l'atto comunicativo su un unico turno informativamente denso. Nel tempo, per queste apprendenti, il progresso si realizza attraverso una graduale distribuzione della richiesta su più turni, dalla bassa densità informativa, con un allineamento nella direzione delle strategie native.

Anche nel corpus di bambini di 8-9 anni, nei casi in cui la richiesta viene introdotta su più turni, non si assiste a una presentazione graduale del nucleo dell'atto; al contrario il turno che precede la richiesta ha lo scopo di aprire il canale di comunicazione con un saluto (es. 15) o richiamare l'attenzione del compagno (es. 16), mentre la richiesta è presentata in modo esplicito e immediato, con un certo grado di complessità strutturale dato dalla combinazione di due tipi di atti e dall'impiego di modificatori morfosintattici:

(15) B: ciao

A: $\quad$ ciao dimmi

B: stamattina non ho mangiato \# ho fame mi potresti preparare un un biscotto

(16) B1: Silvia

B2: sì

B1: mi presteresti la colla \#0_2 l'ho lasciata a casa 
Solo in un caso nel corpus la richiesta viene effettivamente distribuita su più turni che permettono una presentazione esplicita, ma graduale della richiesta. In (17), nel chiedere al genitore del compagno il permesso di invitarlo alla propria festa, il bambino inizia giustificando la richiesta dicendo che sabato è il suo compleanno, passa poi a introdurre il desiderio generico di avere qualche amico presente e solo al terzo turno richiede il diretto coinvolgimento dell'interlocutore:

(17) B: sabato è il mio compleanno

A: sì

B: e vorrei invitare un amico e una amica

A: sì

B: Goffred potrebbe venire al mio compleanno

\section{Analisi qualitativa}

L'analisi qualitativa condotta sui commenti meta-pragmatici realizzati dai bambini durante le attività di riflessione linguistica ha lo scopo di rilevare da un lato come reagiscono gli alunni alle attività didattiche proposte, dall'altro di osservare quale tipo di riflessione possono svolgere a questa età.

La prima parte dell'analisi qualitativa ha permesso di osservare, dalla prospettiva dei bambini, gli aspetti problematici e i punti di forza delle attività proposte. Due gli elementi su cui si concentra l'attenzione in questo contributo: le modalità di ascolto adottate nelle attività di analisi e il lavoro di riflessione linguistica basato sull' analisi delle proprie produzioni.

Rispetto al primo punto - le modalità di ascolto adottate - i dati mostrano come sia complesso per gli alunni, almeno in una fase iniziale, ricordare con precisione le parole impiegate dai compagni nel formulare le richieste. Nelle attività di riflessione linguistica della fase 2 - Utilizzare del percorso infatti, immediatamente dopo la realizzazione di ciascun role play, agli allievi veniva richiesto, oltre che di individuare le variabili sociolinguistiche, di ripetere con precisione l'esatta formulazione linguistica della richiesta. Nello svolgere il compito, i bambini tendono a restituire formule adeguate alla situazione, ma linguisticamente diverse da quelle effettivamente usate dai compagni. Nel riportare in modo poco preciso le parole usate, quasi sempre aggiungono elementi pertinenti ed efficaci, dimostrando così di aver già sviluppato diverse strategie per rendere più cortese o accettabile una richiesta, come esemplificato in (18): il parlante che realizza il role play richiede un libro in biblioteca usando la formulazione vorrei un libro, mentre i compagni, nel cercare di riportare le parole esatte, aggiungono il modificatore per favore, plausibile nella situazione comunicativa proposta, ma non pronunciato realmente dal compagno. 
(18) I: ok e ve la ricordate che cosa aveva detto per chiedere

[...]

B1: per favore

B2: per favore vorrei un libro di tutt-

I: $\quad$ io per favore non l'ho sentito

[...]

B3: non l'ha detto ha detto subito che vuole quel libro

[...]

B4: un libro di tutti \#0_6 che parla

B5: ha detto vorrei un libro per tutto

La difficoltà che emerge nel ripetere con precisione le parole ascoltate sembra essere principalmente dovuta all'abitudine a scuola a intendere l'ascolto quasi esclusivamente come attività di comprensione di contenuto: raramente esso viene proposto come esercizio per l'identificazione attenta delle parole usate da un parlante, come nel caso di questo percorso. Con il procedere dell'attività infatti, e con i ripetuti stimoli del docente, i bambini cominciano a prestare maggiore attenzione alle forme linguistiche e riescono a ripetere l'esatta formulazione usata nel role play dal compagno. Quando l'obiettivo è sviluppare la competenza pragmatica, l'attenzione verso le parole effettivamente utilizzate in una determinata situazione è importante; è una pratica fondamentale per attivare un'efficace riflessione meta-pragmatica e di conseguenza promuovere l'acquisizione di modalità sempre più fini di realizzazione di una richiesta.

Rispetto al secondo aspetto osservato - legato al fatto che il materiale su cui si realizza l'attività linguistica è basato sul parlato degli allievi stessi - i commenti dei bambini mostrano come questa pratica didattica sia stata ampiamente apprezzata e abbia avuto ricadute positive sulla motivazione, così come esemplificato dalla sequenza (19):

(19) I: perché è stato bellissimo

B: perché abbiamo tutti fatto una::: una tipo di azione con le parole

$[\ldots]$

I: poi cos'altro vi è piaciuto

B: spiegare

I: $\quad$ spiegare $\mathrm{mhm}$

B: ci è piaciuto anche sentire gli altri

I: quindi è stato bello anche ascoltare gli altri.

I bambini non sono abituati a trovare nelle loro parole un materiale su cui tornare e su cui condurre attività grammaticale. Questo tipo di esercizio offre invece una serie di vantaggi: non 
solo ha ricadute positive sulla motivazione, ma risulta efficace perché consente di operare sui propri usi linguistici, nel rispetto del livello di competenza di ciascuno.

La seconda parte dell'analisi qualitativa è stata dedicata all'osservazione del tipo di riflessione metalinguistica che sono in grado di compiere i bambini di questa età. L'analisi si è concentrata sia sui commenti meta-pragmatici dopo ogni role play (fase 2 - Utilizzare del percorso didattico) che sulle riflessioni fatte nel corso della lezione dedicata alla riflessione linguistica (fase 3 - Riflettere).

Nella fase 2 - Utilizzare, quando opportunamente guidati, i bambini riescono a riconoscere e spiegare le strategie comunicative utilizzate nei role play, spingendo le loro riflessioni anche su aspetti piuttosto fini della lingua. Ad esempio, nelle sequenze (20) e (21), l'insegnante guida l'osservazione e la riflessione sulle diverse modalità utilizzate per insistere. Nella sequenza (20), il role play di riferimento ha come protagoniste una bambina e la nonna, con la prima che chiede alla seconda il telecomando per cambiare canale e guardare il suo programma preferito. Di fronte al rifiuto della nonna, la bambina insiste limitandosi a ripetere la stessa richiesta e mantenendo l'identica formulazione - posso cambiare canale? -, senza però riuscire a ottenere una risposta positiva. Durante la riflessione in gruppo, l'insegnante guida i bambini a elencare possibili strategie che possono rendere l'azione dell'insistere più efficace: si sofferma infatti non solo sulle parole utilizzate dalla bambina, ma richiama l'attenzione sul numero di volte in cui la richiesta è stata ripetuta, portando la classe a notare come in una situazione simile il richiedente poteva più efficacemente variare la richiesta, combinando ad esempio più tipi di atti, introducendola con una Giustificazione, o modificandone la forza illocutoria:

(20) I: nonna e nipotina \#0_8 cosa chiede quando \#0_6 le domande di richiesta non le risposte

B1: posso cambiare canale

I: lo ripete solo una volta

CL: noo

B2: due

CL: noo tre

B3: mille

I: lei ha chiesto posso cambiare canale \#0_2 se la mamma o la nonna vi dicono in questo momento di no voi continuate a fare la stessa domanda. \# posso cambiare canale posso cambiare canale posso cambiare canale \#0_2 o dite un'altra cosa o dite delle altre cose

B4: altre cose

I: per esempio che cosa dici

B5: gli dico: \#0_2 visto che io voglio \#0_4 vorrei assolutamente guardare la televisione I: ok però fai sempre la stessa domanda oppure provi a cambiare 
B6: provo a cambiare

B7: provo a chiedere \#0_2 mh: potrei mettere su un altro canale \#0_2 perché questo \#

B8: due secondi

I: $\quad$ per due secondi \#0_4 oppure se proprio in quel momento c'è il vostro cartone animato preferito e lo danno solo a quell'ora lì

B: quando finisce mi chiami che guardo io un po' la televisione

Nel commento (21) invece l'interazione stimolo è ambientata in palestra, ha come protagoniste una bambina e il suo allenatore e prevede che la bambina chieda all'allenatore di poter giocare a pallasqualo. Di fronte al rifiuto iniziale, la bambina prosegue l'interazione rilanciando la richiesta e insistendo. In gruppo la discussione si concentra sulle parole utilizzate per insistere in modo cortese (solo per questa volta, ti prego). I bambini in autonomia confrontano l'episodio con l'interazione precedente e spiegano le ragioni che hanno portato a esiti diversi:

(21) B: come quello: posso cambiare canale per due secondi:

\#0_2 solo per questa volta è una richiesta così aveva più possibilità che gli dicesse di sì perché se uno dice per tutto il tempo possiamo giocare a pallasqualo allora gli diceva di no ma se gli dice solo per questa volta.

La terza parte dell' analisi qualitativa ha tenuto conto delle osservazioni emerse nella fase 3 - Riflessione del percorso didattico in cui l'insegnante, a partire da attività di approfondimento strutturate, aiuta i bambini a riflettere ulteriormente sulle diverse modalità di formulazione di una richiesta e sulle possibili variazioni linguistiche legate al contesto comunicativo, all'oggetto da richiedere e all'interlocutore. Una delle prime osservazioni che emergono è che le richieste si possono realizzare in modo differente, così come esemplificato dal commento (22) di uno degli allievi:

(22) B: ci sono tanti diversi modi per fare una richiesta a volte sono frasi molto lunghe altre bastano poche parole.

Poiché i bambini hanno capito che esistono molti modi per fare una stessa richiesta, l'insegnante approfondisce ulteriormente la riflessione adottando un cambio di prospettiva: nella sequenza (23), fa notare come una stessa richiesta possa essere realizzata in situazioni e contesti diversi, proponendo la formulazione - si potrebbe avere una sedia? - e chiedendo ai bambini di individuare in quali luoghi potrebbero sentire questa frase, compito che gli allievi compiono senza difficoltà:

(23) B1: in pizzeria [...] perché se manca una sedia in pizzeria 
B2: al ristorante [...] quando sei al tavolo e.. manca una sedia

B3: al supermercato perché se tu stai comprando una sedia o in un negozio

B4: a casa di un mio amico

B6: tipo in teatro [...] perché se manca una sedia

Successivamente, l'insegnante stimola le variazioni di formulazione proponendo l'uso di modificatori o atti di supporto, ricollegando le diverse possibilità a variabili sociopragmatiche, con un continuo collegamento bidirezionale tra forma e funzione. Come è possibile osservare in (24), la riflessione si svolge senza fornire etichette e definizioni che risulterebbero per questi allievi troppo astratte e poco funzionali:

(24) I: adesso facciamo la cosa al contrario, se io voglio dire questa stessa cosa voglio fare questa stessa richiesta con altre parole

B1: maestra manca una sedia potrebbe portarla

B2: maestra posso prendere una sedia

I: allora. [...] maestra posso prendere una sedia ancora altri modi per chiedere la stessa cosa invece che scusi non si potrebbe.. [nome] come diresti tu come chiedi se vuoi avere una sedia

B3: scusi posso avere una sedia

B4: se no per favore posso avere una sedia

B3: scusi mi dà una sedia

$[\ldots]$

B2: anche potreste

I: potreste potrei avere una sedia allora.. ultima domanda..e adesso E. mi aiuta. Mi dici secondo te che differenza c'è tra questa prima frase sia che io dica maestra, mamma, signore posso prendere una sedia posso avere una sedia per favore potreste darmi una sedia mi dà una sedia, potrei avere una sedia \#0_4 secondo voi si possono usare con tutti alla stessa maniera queste frasi oppure scegliete una a seconda di che cosa?

B4: il posto dove sei

$[\ldots]$

B3: scusi signora mi potrebbe dare una sedia

I: $\quad$ mi potrebbe dare una sedia se è una signora che non conosci se invece vai a casa di un amico e la mamma la conosci

B3: dici.. mi potresti passare una sedia

$[\ldots]$

B1: tipo nonna per piacere mi passi la sedia 
L'introduzione di questo tipo di attività in classe è un utile strumento di ripresa e rinforzo di una parte della terminologia metalinguistica sviluppata in attività precedenti. Nella pratica più tradizionale spesso gli argomenti grammaticali, dopo essere stati trattati, vengono dati più o meno per acquisiti e archiviati, per passare a un altro argomento (MIGLIETTA; SOBRERO, 2011). L'analisi delle strategie utilizzate dai parlanti nelle diverse situazioni comunicative permette invece alle insegnanti di creare un collegamento con le attività di descrizione delle strutture grammaticali e riprendere da una diversa prospettiva alcune strutture rilevanti. L'importanza di esercitare esplicitamente l'abilità di saper riconoscere un elemento a partire dall'uso, e di riuscire a individuarne la funzione in un determinato contesto, anche senza etichettarlo con un meta-linguaggio astratto, emerge chiaramente anche dai commenti degli insegnanti durante gli incontri di formazione. Ecco come una di loro descrive il processo di riflessione attiva sulla grammatica in uso:

Quando ne abbiamo parlato insieme la prima cosa che hanno detto i bambini è: "qui maestra stiamo usando il condizionale" e abbiamo proprio fatto una riflessione e abbiamo proprio ripreso il condizionale per riuscire a ricordare la differenza tra condizionale e indicativo e perché lo stavamo utilizzando in questo contesto. Altri hanno notato subito che in alcune frasi delle scenette ascoltate non c'era neanche il verbo quindi era molto immediato riuscire a prendere/chiedere il caffè anzi "perché tanto al bar se non vai a prendere un caffè che cosa vai a prendere?" a casa dell'amico invece "scusami guarda guarda sono stanchissimo un po' di caffè" Quindi anche motivare fare capire dare una motivazione.

Come recentemente sottolineato in un intervento da Pistolesi (2017), riprendendo un'idea già espressa in passato da Colombo (1997), "non si può fare un'equazione diretta tra la regola, lo studio della regola, l'aver appreso la regola e saperla mettere in pratica". L'attività di riflessione meta-pragmatica, messa in atto grazie alle sollecitazioni del docente a notare fenomeni e a individuare in modo collettivo spiegazioni della funzione di determinate scelte linguistiche rispetto ad altre, sembra riuscire a generare utilissime 'spinte' all'avanzamento della competenza comunicativa e allo sviluppo di una maggiore consapevolezza, così come sottolineato anche dalle parole di un docente in (26):

(26) C'è stato proprio un bambino che ha detto "ho capito che cosa stiamo poi facendo in questi giorni" perché erano un po' straniti da noi "stiamo imparando a capire a che serve a fare riflessione linguistica". Che insomma io dico sempre: bambini questa riflessione che facciamo è utilissima anzi, anche quella testuale. In realtà è la pratica che fa capire l'utilità di tutto quello che viene fatto". 
In altre parole, la riflessione linguistica intesa come attività che porta i bambini a individuare i rapporti tra forma e funzione prima, e solo successivamente, ove necessario, introduce il meta-linguaggio specifico per riflettere più consapevolmente sulla comunicazione, porta a sviluppare nella pratica didattica una consapevole attenzione verso la lingua, le sue regole e i suoi usi, favorendo un più efficace sviluppo della competenza linguistico-comunicativa.

\section{Conclusioni}

Nel presente contributo si è presentato un primo studio dedicato all'esplorazione delle modalità di uso della lingua orale in situazioni interattive di richiesta da parte di bambini della classe terza e quarta della scuola primaria. L'analisi quantitativa ha messo in luce alcune tendenze generali rispetto alle modalità di realizzazione e di gestione di tale atto comunicativo nell'interazione orale, con pari e adulti. La lettura dei dati ha permesso di rilevare come $\mathrm{i}$ bambini di questa età sappiano già utilizzare diversi tipi di atti e dunque variare con sufficiente competenza le loro modalità di realizzazione dell'atto comunicativo, nonostante, nel complesso, le loro richieste siano ancora strutturare in modo semplice e diretto. Essi devono pertanto imparare a introdurre gradualmente il nucleo dell'atto comunicativo attraverso la combinazione di diversi atti e l'uso di una più ampia gamma di modificatori, mentre dal punto di vista interazionale devono ancora apprendere a gestire una più fluida negoziazione della richiesta con l'interlocutore, attraverso l'uso di una maggiore varietà di atti di supporto e una più efficace distribuzione dell'atto stesso su diversi turni interazionali. I bambini hanno dunque già sviluppato una serie di competenze, che possono essere ulteriormente rafforzate attraverso un insegnamento esplicito. L'analisi qualitativa delle riflessioni meta-pragmatiche emerse spontaneamente o stimolate dal docente nelle attività dedicate all'osservazione linguistica ha infatti evidenziato proprio come i bambini, quando opportunamente guidati, riescono a notare fenomeni anche complessi e a riflettere sul rapporto tra variabili sociopragmatiche e variazione linguistica. Per utilizzare le parole di una delle docenti coinvolte nella sperimentazione "l'ingresso della pragmatica in aula è stata una nuova finestra spalancata sulla lingua stessa e sulle sue norme che i bambini usano da sempre, ma senza consapevolezza a volte dei vincoli, delle sfumature, delle opportunità che offre e che pretende da chi le parla".

Dal punto di vista didattico, il presente studio offre diversi spunti di riflessione. Innanzitutto, l'analisi quantitativa evidenzia quali siano i reali bisogni educativi nei bambini di terza e quarta elementare: non si tratta tanto di imparare a fare una richiesta, ma piuttosto, come abbiamo già detto sopra, di sviluppare la consapevolezza metacomunicativa e migliorare l'uso delle strategie necessarie per mitigare l'atto e gestirlo efficacemente nell'interazione. I materiali didattici che possono costituire la continuazione di questa esperienza, anziché basarsi su una intuizione generica del docente, vengono definiti così dall'osservazione precisa di ciò che c'è e 
di ciò su cui si può opportunamente lavorare in classe per uno sviluppo articolato delle abilità di uso linguistico. In secondo luogo, i commenti meta-pragmatici di bambini e insegnanti, rafforzano l'idea alla base del progetto, ovvero l'importanza di un'attenzione specifica alla lingua orale e ai suoi usi a scuola: questa pratica non solo è motivante e con una chiara ricaduta sulle abilità di interazione orale per sé, ma soprattutto favorisce lo sviluppo di competenze nella riflessione linguistica da parte dei bambini, qui intesa appunto come osservazione della lingua in uso e conseguente individuazione di relazioni tra forma e funzione.

Il tipo di attività grammaticali proposte stimola infatti i bambini a osservare e comprendere gli effetti sulla comunicazione della variazione linguistica. Questo promuove, oltre a una maggiore consapevolezza meta-pragmatica, lo sviluppo delle competenze linguisticocomunicative, risultando di conseguenza più efficace nel far progredire le abilità d'uso degli allievi rispetto alla più tradizionale analisi grammaticale, logica o del periodo, concepita invece come mera capacità di identificare e denominare in modo astratto e meccanico le strutture linguistiche (cfr. LO DUCA, 2003; PALLOTTI 2009). Pragmatica e grammatica in questo senso dialogano tra loro nella pratica didattica, così come nell'uso reale della lingua, e a partire da percorsi bidirezionali, che vanno dalla pragmatica alla grammatica e viceversa, si favorisce lo sviluppo delle capacità di uso, in stretta relazione con le capacità di riflettere sugli usi stessi, portando i bambini a selezionare le strategie più adeguate nei vari contesti situazionali. Infine, permettere ai bambini di riflettere sulla lingua da loro prodotta e sui suoi usi più quotidiani non solo ha un impatto positivo sulla motivazione ad apprendere, ma favorisce anche la partecipazione di tutti gli allievi, indipendentemente dal loro livello di competenza linguistica. Sulla base della sperimentazione condotta - per quanto circoscritta ad alcune classi scolastiche - si può affermare che una maggior attenzione alla componente pragmatica nell'ambito dell'educazione linguistica non possa essere relegata a un progetto estemporaneo o limitato nel tempo, ma auspicabilmente debba diventare una pratica integrata nella programmazione, fin dalla scuola primaria.

Dal punto di vista della ricerca, il presente studio mette in luce alcuni punti di attenzione che possono essere meglio indagati. Poiché la ricerca è stata inserita in un percorso di ricercaazione, non è stato possibile controllare con precisione tutte le variabili, ad esempio ottenere uno stesso numero di produzioni per i due tipi di richieste o di interlocutori. Uno studio sperimentale risolverebbe tale lacuna. Nel complesso, sarebbe utile definire futuri protocolli di ricerca che permettano di osservare una maggior tipologia di tipi di richieste, con un controllo della variabile richiesta più o meno impegnativa, oltre che indagare lo sviluppo della competenza pragmatica nel tempo, raccogliendo un corpus di dati longitudinali. 


\section{Riferimenti bibliografici}

ANDORNO, C. Che cos'è la pragmatica linguistica. Roma, Carocci, 2005.

BAZZANELLA, C. Le facce del parlare. Firenze, La Nuova Italia, 1994.

BAZZANELLA, C. Linguistica e pragmatica del linguaggio. Roma-Bari, Laterza, 2005.

BETTONI, C. Usare un'altra lingua. Guida alla pragmatica interculturale. Roma-Bari, Laterza, 2006.

COLOMBO, A. Per un' "educazione linguistica essenziale": la riflessione sulla lingua. La didattica, III, 3 marzo 1997, pp. 51-5.

FERRARA, A. Pragmatics. In Van Dijk, T. (ed.) Handbook of discourse analysis. London, Academic Press, 2, 1985, pp. 137-57.

FERRARI, S. Elementi di variazione della complessità sintattica. In Consani, C.; Desideri, P. (a cura di). Alloglossie e comunità alloglotte nell'Italia contemporanea, Atti del XLI Congresso Internazionale di Studi della Società di Linguistica Italiana, Pescara, 27-29 settembre 2007. Roma, Bulzoni, 2009, pp. 155-70.

FERRARI, S. A Longitudinal Study of Second Language Learner Complexity, Accuracy and Fluency Development. In Housen, A.; Kuiken, F.; Vedder, I. (eds.) Dimensions of L2 Performance and Proficiency: Investigating Complexity, Accuracy and Fluency in SLA. Amsterdam: Benjamins, 2012, pp. 277-97.

FERRARI, S. Oggi facciamo pragmatica: un progetto di formazione e ricerca-azione nella scuola primaria. Italiano LinguaDue, 8, 2, 2016, pp. 270-80.

FERRARI, S.; NUZZO, E., Un'osservazione longitudinale sul rapporto tra pragmatica e grammatica nell'acquisizione dell'italiano L2. In Bozzone Costa, R.; Fumagalli, L.; Valentini, A. (a cura di) Apprendere l'italiano da lingue lontane: prospettiva linguistica, pragmatica, educativa. Atti del Convegno-Seminario Bergamo, 17-19 giugno 2010. Perugia, Guerra Edizioni, 2011, pp. 159-72.

FERRARI, S.; NUZZO, E.; ZANONI, G. Sviluppare le competenze pragmatiche in L2 in rete: problemi teorici e soluzioni pratiche nella progettazione dell'ambiente multimediale LIRA. In Cervini, C. (a cura di) Interdisciplinarità e apprendimento linguistico nei nuovi contesti formativi. L'apprendente di lingue tra tradizione e innovazione. Bologna, Quaderni del CeSLiC - Centro di Studi LinguisticoCulturali, 2016, pp. 5-20.

LEONE, P.; MEZZI, T. Didattica della comunicazione orale. Milano, Franco Angeli, 2011.

LO DUCA, M. G. Lingua italiana ed educazione linguistica. Roma, Carocci, 2003.

MATTHEWS, D. (eds.), Pragmatic Development in First Language Acquisition. AmsterdamPhiladelphia, Benjamins, 2014.

MIGLIETTA, A.; SOBRERO, A. Pratiche di grammatica nella scuola elementare: un'indagine. In Corrà L.; Paschetto W. (a cura di) Grammatica a scuola. Milano, Franco Angeli, 2011, pp. 97-112.

NUZZO, E. Imparare a fare cose con le parole. Perugia, Guerra Edizioni, 2007.

NUZZO, E. Imparare ad agire in italiano L2: uno studio con giovani immigrate. In Nuzzo, E.; Gauci, P. Insegnare la pragmatica in italiano L2. Roma, Carocci Editore, 2012, pp.45-74. 
NUZZO, E. Fonti di input per l'insegnamento della pragmatica in italiano L2: riflessioni a partire dal confronto tra manuali didattici, serie televisive e parlato spontaneo. In Santoro, E.; Vedder, I. (a cura di) Pragmatica e interculturalità in italiano lingua seconda. Firenze, Franco Cesati Editore, 2016, pp. 15-28.

NUZZO, E.; GAUCI, P. Insegnare la pragmatica in italiano L2. Roma, Carocci Editore, 2012.

OLSHTAIN, E.; COHEN, A. Apology: A speech act set. In Wolfson, N.; Judd, E. (eds.) Sociolinguistics and language acquisition. Rowley, Newbury House, 1983, pp. 18-36.

PALLOTTI, G. Descrivere le lingue: quale metalinguaggio per un' educazione linguistica efficace? In PROGETTO PON Educazione Linguistica e Letteraria in un'Ottica Plurilingue, MIUR/ANSAS, 2009,

http://www.scuolavalore.indire.it/nuove_risorse/descrivere-le-lingue-quale-metalinguaggio-per-uneducazione-linguistica-efficace/. Ultimo accesso: 15 aprile 2017.

PALLOTTI, G.; FERRARI, S. La variabilità situazionale dell'interlingua: implicazioni per la ricerca acquisizionale e il testing linguistico. In Bernini, G.; Spreafico, L.; Valentini, A. (a cura di) Competenze lessicali e discorsive nell'acquisizione di lingue seconde. Perugia, Guerra, 2008, pp. 437-62.

PALLOTTI, G.; FERRARI, S.; NUZZO, E.; BETTONI, C. Una procedura sistematica per osservare la variabilità nelle interlingue. Studi Italiani di Linguistica Teorica e Applicata, 39, 2, 2010, pp. 215-41.

PISTOLESI, E. L'italiano nei libri di testo. Comunicazione presso l'Accademia dei Lincei e Scuola Normale Superiore, 3 febbraio 2017, https://www.youtube.com/watch?v=vW-NSwoC7mM. Ultimo accesso: 10 aprile 2017.

SANTORO, E.; VEDDER, I. Pragmatica e interculturalità in italiano lingua seconda. Firenze, Franco Cesati Editore, 2016.

TROSBORG, A. Interlanguage pragmatics. Requests, Complaints and Apologies. Berlin-New York, Mouton de Gruyter, 1995.

Recebido em 24/05/2017

Aprovado em 15/10/2017 